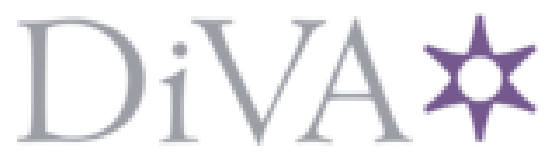

http://www.diva-portal.org

This is the published version of a paper published in IEEE Sensors Journal.

Citation for the original published paper (version of record):

Rydblom, S., Thörnberg, B. (2016)

Liquid Water Content and Droplet Sizing Shadowgraph Measuring System for Wind Turbine Icing Detection.

IEEE Sensors Journal, 16(8): 2714-2725

http://dx.doi.org/10.1109/JSEN.2016.2518653

Access to the published version may require subscription.

N.B. When citing this work, cite the original published paper.

Permanent link to this version:

http://urn.kb.se/resolve?urn=urn:nbn:se:miun:diva-2732 1 


\title{
Liquid Water Content and Droplet Sizing Shadowgraph Measuring System for Wind Turbine Icing Detection
}

\author{
Staffan Rydblom and Benny Thörnberg
}

\begin{abstract}
This paper shows that the liquid water content (LWC) and the median volume diameter (MVD) can be derived from the images of water droplets using a shadowgraph imaging system with incoherent LED illumination. Icing on structures, such as a wind turbine, is the result of a combination of LWC and MVD, and other parameters, such as temperature, humidity, and wind speed. Today, LWC and MVD are not commonly measured for wind turbines. Systems for measuring these properties are often expensive or impractical in terms of location or remote reading. The aim of this paper is to gain knowledge about how to design a single instrument based on imaging that has the ability to measure these properties with enough precision and accuracy to detect icing conditions for wind turbines. A method to calculate both the LWC and the MVD from the same images is described in this paper. The size of one droplet is determined by measuring the shadow created by the droplet in background illumination. The concentration is calculated by counting the measured droplets and estimating the volumes in which these droplets can be observed. In this paper, the observation volume is shown to be dependent on the particle size and the signal-to-noise ratio for each measured particle. An expected coefficient of variation of the LWC depending on the droplet size is shown to be $2.4 \%$ for droplets $10 \mu \mathrm{m}$ in diameter and $1.6 \%$ for $25-\mu \mathrm{m}$ droplets. This is based on an error estimation of the laboratory measurements calibrated using a micrometer dot scale.
\end{abstract}

Index Terms-Clouds, image processing, machine vision, meteorology, optical microscopy, wind power generation.

\section{INTRODUCTION}

I CING caused by freezing atmospheric water is a problem for the generation of electrical energy from wind in cold climates. Unless prevented, it may lead to lower performance or to the wind turbines stopping completely [1]-[4]. Aircraft, power lines or any other weather exposed structure share this problem. Therefore big efforts have been made to create models for how the ice is formed [5], [6] and how it can be included in weather prediction models [7], [8].

The icing process is complex and the result depends on a combination of the aerodynamic shape of the structure or airfoil, the velocity of the air and its contained water, the temperature, the mixing of snow and water, the concentration of liquid water and the droplet size distribution. All droplets or particles passing the airfoil are affected by a force

Manuscript received November 25, 2015; revised January 12, 2016; accepted January 12, 2016. Date of publication January 18, 2016; date of current version February 24, 2016. This work was supported by Energimyndigheten. The associate editor coordinating the review of this paper and approving it for publication was Prof. Bernhard Jakoby.

The authors are with Mid Sweden University, Sundsvall 851 70, Sweden (e-mail: staffan.rydblom@miun.se; benny.thornberg@miun.se).

Digital Object Identifier 10.1109/JSEN.2016.2518653 created by the change in pressure surrounding the airfoil. To hit the surface and take part in the icing process they must have enough mass and density. Smaller droplets and less dense particles are pushed into a diverting, non-impact trajectory [5]. The liquid water content (LWC) and the water droplets median volume diameter (MVD) are essential input parameters to predict or model icing. Although there do exist methods, as we will discuss, to measure these properties, they are scarcely ever measured at a planned or existing wind turbine [9], [10]. Measuring them accurately and frequently would be an advantage for the planning of new wind mill farms or for the application of anti-icing arrangements on existing power stations. It may be of particular interest as input to weather prediction models by which both LWC and MVD can be computed [7], [8]. In combination with information about the aerodynamic properties of the wind turbine, it can give more accurate predictions of icing or even result in better design of wind turbines and anti-icing methods.

While icing caused by large supercooled droplets, with diameters from approximately $50 \mu \mathrm{m}$ to exceeding $1000 \mu \mathrm{m}$, is often considered severe due to its shape and quick build-up, icing may occur even with droplets as small as $5 \mu \mathrm{m}$ [11]-[13]. In most cases, though, icing is caused by cloud droplets measuring between $10 \mu \mathrm{m}$ and $30 \mu \mathrm{m}$ in diameter [10], [12].

Although optical imaging and other techniques for measuring aerosol properties is continuously improving, the choice of instrument is still very much dependent on the application's requirements [14]-[17]. An instrument for measuring icing parameters for wind turbines should be able to detect supercooled cloud droplets from five micrometer and determine an accurate measure of the LWC. Since measurements are needed in multiple remote locations it should also be affordable, reliable, have low power consumption, and ideally be possible to place near the highest point of the turbine [18].

\section{A. Contributions}

We describe a shadowgraph system consisting of a monochrome digital image sensor with a telecentric lens and a collimated LED illumination. A method for segmentation of objects from image background, based on the second derivate of the intensity is proposed.

We show that calibration of true droplet size and measuring range both depends on the measured size of the droplet shadow and the amount of light used for exposure. We show that it is possible to predict both the precision of the measured size and accuracy for measurement of droplet size. 
A value of both MVD and LWC can be derived from a series of images and since the number of measured droplets will depend on the concentration, the accuracy and precision will depend on the number of samples from the total population of droplets.

Many existing instruments suffer from errors caused by the instrument itself during sampling, e.g. when droplets get stuck on the inlet [19], or shatters into smaller droplets [20]. An instrument should be designed in order to affect the free flow of particles as little as possible [16]. Therefore we also investigate the illuminative power required to get a good exposure with the tested system at a targeted maximum wind speed of $50 \mathrm{~m} / \mathrm{s}$.

In this study, we have used a stage micrometer scale for characterization of the system and simulation of water droplets. This characterization holds true given that the optical silhouette of a droplet is comparable to a dot having equal diameter and being printed on a silicon glass. It is not a new concept and has at least once been proved experimentally for coherent light, by comparing with beads of glass and water droplets of known sizes [21]. The shadow image of water drops of any size will be defined mainly by the diffracted component, as long as the distance between the drop and the lens is much larger than the drop diameter. Only in a small bright spot in the middle will the refracted component be large enough to be visible in a shadowgraph system [21], [22].

Using the results of this study, a weather protected prototype may be built to perform a comparative study.

We believe that this study of a shadowgraph imaging system provides good analysis of its expected major limitations related to the measurement of liquid water content of air. This is also the scientific contribution of this publication.

\section{B. Related Work}

In 1970, Knollenberg [23] described an electro-optical technique to measure cloud and precipitation particles using a laser illuminated linear array of photo detectors. The photo detectors are used to make a two-dimensional image of the particles' shadows as they pass the light beam. Systems based on this technique are called Optical Array Probes (OAP) or two-dimensional imaging probes. Later development of this technique includes using image sensors to save gray scale images of the detected particles [17], [22].

One popular technique to measure single particles used in several instruments is to measure the light scattered from a focused laser [15], [24]. A laser beam is used to illuminate passing particles. When a particle is detected, its size is determined by comparing the variations in light with a pattern calculated from the Mie scattering theory [25].

Since the OAP, its successors and the scattering spectrometers have advantages over the other depending on the size and nature of the aerosol, instruments for airborne use have been developed that combine several techniques into one single probe [26] for accurate measuring of LWC and MVD [16].

A different optical technique for measuring water droplets is based on in line holography [27]. In principle this is a two-dimensional shadowgraph imaging system that use laser

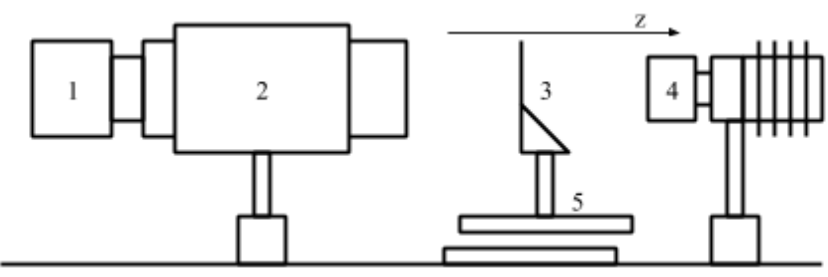

Fig. 1. The optical setup: 1) camera, 2) Lens, 3) Holder for micrometer scale, 4) LED with collimating lens, and 5) Translation stage.

background illumination to create images of the diffraction patterns created by the passing particles. These patterns are measured to reconstruct images of the particles. This is a fairly calculation intensive process, which may be one of the reasons why instruments based on this technique are not so common [28].

Sizing of the droplets using Mie spectrometry is a complex operation even for coherent light [29], and although it is possible to study Mie scattering from white light [30], the complexity, small droplet size range and sample volume makes it less attractive in this application. The optical resolution is usually too low. Therefore it is also difficult to determine the exact particle size and the usable depth of field for incoherent shadowgraph systems. The shadow from a particle can e.g. appear smaller or larger when out of focus.

Shadowgraph imaging of particles using incoherent illumination instead of laser has been tried e.g. in particle shadow velocimetry (PSV) [31], [32], or spray characterization [33]. Quantitative and comprehensive studies of other droplet measurement techniques exist [24], [28], [34]. But to our knowledge there is no publication providing an equal analysis of a shadowgraph system using incoherent light for droplet measurement. We believe this study provides a good base for the design of a shadowgraph system for particle measurement using incoherent illumination.

\section{Materials AND Methods}

The experimental setup is illustrated in Fig. 1. The camera (1) is mounted on a telecentric lens (2) and fixed in line with a holder for a test object (3) and the backlight illumination (4). The object holder was mounted on a translation stage (5) to move the object in the z-direction.

\section{A. Materials and Methods}

The camera used throughout this study was the uEye UI-3370CP-NIR. It contains a monochrome CMOS digital image sensor, CMOSIS CMV4000, with $12 \mu \mathrm{m}$ epilayer for improved spectral response in the near infrared (700 to $900 \mathrm{~nm}$ ). The sensor has $2048 \times 2048$ active pixels on a $5.5 \mu \mathrm{m}$ pitch. The image array has pipelined global shutter pixels which allow exposure during read out, while performing CDS (correlated double sampling) operation. The image sensor has sixteen 10- or 12-bit serial outputs. It also integrates a programmable gain amplifier and offset regulation. Each channel runs at $480 \mathrm{Mbps}$ maximum which results in $180 \mathrm{fps}$ frame rate at full resolution (10-bit mode). 
The background illumination was produced using an LED, Mightex LCS-0455-03, with a $22 \mathrm{~mm}$ collimating lens. The LED produces low-coherent light in a spectra with its peak intensity at $455 \mathrm{~nm}$ (blue). The $22 \mathrm{~mm}$ lens produced a square beam approximately 8 times $8 \mathrm{~mm}$. According to Mightex, the half diverging angle using $22 \mathrm{~mm}$ aperture is 1.7 degrees.

The lens was a telecentric lens, Opto Engineering TC4M004-C, with $4.04 \times$ magnification according to the delivery test report. With the described camera it gives an object field of view of 2.79 times $2.79 \mathrm{~mm}$. The working f/\# is 22 and the depth of field is given to approximately $0.1 \mathrm{~mm}$. Depth of field is discussed more extensively in chapter IV. The given lens was tested at delivery for telecentricity in four image zones, from the center to the edge, giving a telecentric slope from 0.015 and 0.091 degrees. This means that the maximum error for a displacement of $1 \mathrm{~mm}$ would be less than 1.5 micron in a corner of the lens, and less than 0.3 micron in the center.

A stage micrometer scale, Edmund Optics \#58-606, with dots printed in chrome on a silicon glass was used as reference object. The pattern scale featured printed dots in diameters of $2 \mu \mathrm{m}, 3 \mu \mathrm{m}, 4 \mu \mathrm{m}, 5 \mu \mathrm{m}, 6 \mu \mathrm{m}, 7 \mu \mathrm{m}, 8 \mu \mathrm{m}, 9 \mu \mathrm{m}, 10 \mu \mathrm{m}$, $25 \mu \mathrm{m}, 50 \mu \mathrm{m}, 75 \mu \mathrm{m}$ and $100 \mu \mathrm{m}$. It was mounted on a translation stage that was used to automatically move the object in steps of two micrometers between the images. The distance between each step is called the delta distance $\Delta z$. See Fig. 5. The measuring range corresponds to the delta distance at which the reference object could be detected using the proposed method for image segmentation.

\section{B. Image Edge Detection}

The reason for using edge detection is that the edge response can be used both for measuring the size of the droplets and to define the measurement volume. Droplets with too weak edge response would be outside the measurement volume.

In this study we have used a Laplacian of Gaussian [35] edge detection filter implemented as a $13 \times 13$ size convolution kernel. This method is attractive in digital image processing applications since the Laplacian derivation and the Gaussian blurring filter can be combined in a single convolution kernel, thus reducing the necessary number of multiplications to process a complete image. Blurring before derivation is needed to reduce the sensitivity to noise in the image. The result is an image of the second order derivative that is near zero very close to an edge and in regions far away from an edge. The Laplacian for a two dimensional function (image) $f(x, y)$ is defined in $\mathrm{Eq} 1$.

$$
\nabla^{2} f=\frac{\delta^{2} f}{\delta x^{2}}+\frac{\delta^{2} f}{\delta y^{2}}
$$

The second order derivate is positive on one side of the edge and negative on the other side [36]. We look for zero crossings in the $\mathrm{x}$ - and $\mathrm{y}$-directions of the filtered image, i.e. where the value is negative/positive on one side and positive/negative on the other. A threshold is set to the absolute value of the difference between the negative and positive value greater than zero and these crossings are marked in a new image. The result
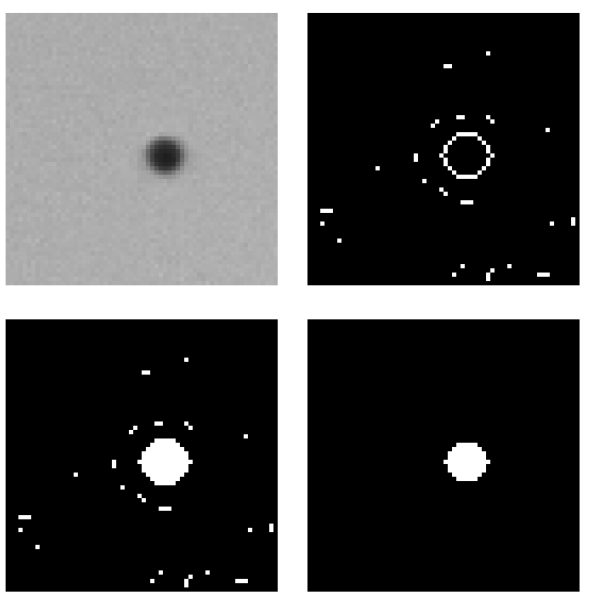

Fig. 2. Ten micrometer dot (upper left), edge detected (upper right), filled (lower left) and substracted to a single blob (lower right).

is a binary image with contours marking the objects that have sharp edges. See Fig 2, upper right.

\section{Object Identification}

Using the edge detected binary image, we apply a boundary fill function (Fig. 2, lower left). The boundary fill detects closed contours in binary images like the one shown (Fig. 2, upper right), that are not in contact with the edge of the image and alters the value of the pixels within the boundary. The filled boundaries are identified as objects to be measured by subtracting the filled image with the original edge binary (Fig. 2, lower right).

\section{Object Size Measurement}

The cross sectional area of each identified object is determined by counting the number of pixels in its filled boundary. From the cross sectional area we calculate the uncorrected diameter by using the magnification of the lens and the pixel size of the image sensor.

\section{E. Measuring Signal to Noise Ratio}

The noise in an image is the non-deterministic modulation $\sigma_{j}$ stemming from photon quantization, thermal energy, spatial sensitivity variations and more. Its magnitude depends both on the camera sensor's SNR and the variation in intensity of the light beam. In particular the intensity may differ from one image to another, depending on e.g. the LED temperature, the length and power of the electrical pulse and external disturbances in the optical path. Therefore we construct a method to calculate the signal to noise ratio $\left(\mathrm{snr}_{j}\right)$ for every measured particle $j$. The noise $\sigma_{j}$ is measured from the background signal around each particle $j$. The signal is the impact, or the amplitude, $\mathrm{A}_{j}$, of the shadows caused by the drop. The snr is the relation between the signal and the noise.

$$
\operatorname{snr}_{j}=20 \log \frac{\mathrm{A}_{j}}{\sigma_{j}}
$$




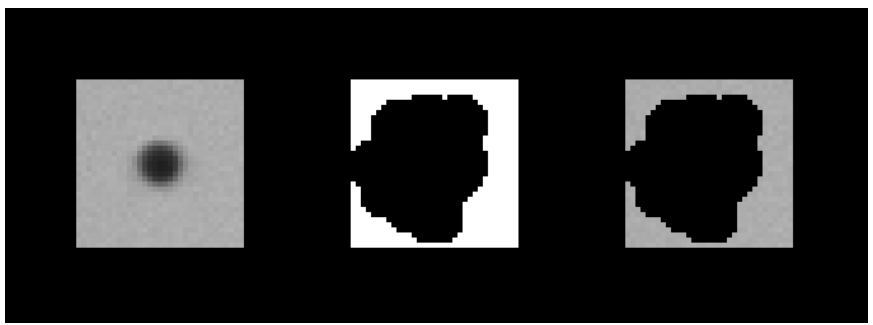

Fig. 3. Background noise. The image is cropped to a square (left) around the particle $j$ (arrowed). The size of the square depends on the size of the particle. A black mask (middle) is calculated to get the background noise signal (right) containing all $x_{i, j}$ for particle $j$.

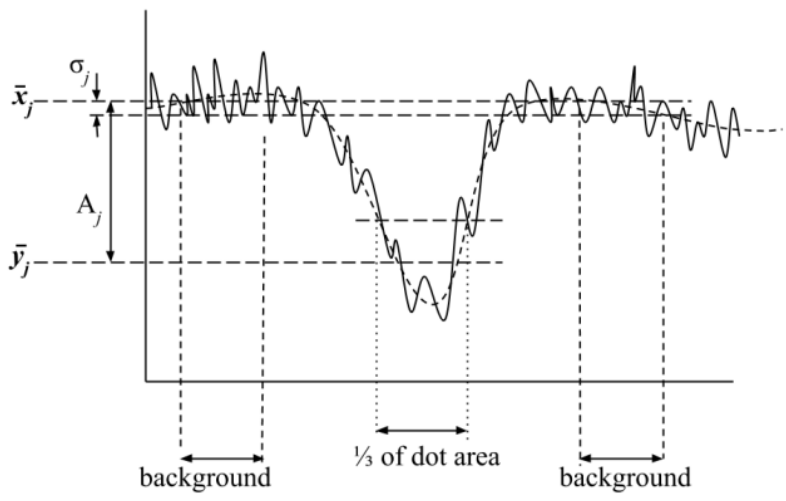

Fig. 4. SNR calculation. $\bar{y}_{j}$ is determined by calculating the average of the darkest third of all pixels inside the edge detected dot.

where

$$
\mathrm{A}_{j}=\left|\bar{x}_{j}-\bar{y}_{j}\right|
$$

and

$$
\sigma_{j}=\sqrt{\frac{1}{N} \sum_{i=1}^{N}\left(x_{i, j}-\bar{x}_{j}\right)^{2}}
$$

$\mathrm{A}_{j}$ is the difference between $\bar{x}_{j}$ and $\bar{y}_{j} \cdot \bar{y}_{j}$ is the mean of one third of the smallest values in the area covered by the filled blob constructed from the edge detection. See illustrations in Fig. 2, and Fig. 4. $x_{i, j}$ is each of the $\mathrm{N}$ pixel values of the background noise surrounding particle $j$. To exclude pixels that may be affected by the diffraction from the light passing, we used a five pixel large disk shaped structure to dilate the original boundary. Therefore the pixels immediately next to the edge-detected boundary are not counted in the SNR calculation. $\bar{x}_{j}$ is the mean of $x_{i, j}$ for each particle $j$. See Fig. 3 and Fig. 4.

The background is limited to an equal-sided square surrounding each measured object with the side equal to the size of the edge detected multiplied by two, plus ten pixels.

Defining the SNR in this way makes it possible to determine an individual SNR for every measured object in an image. True size and detection range can then be calibrated depending on its measured size and SNR.

\section{F. Creating Calibration Tables}

A translation stage is used to move the object in steps from closer than in focus to further away than the focus of the lens. All the dot images are then analyzed using the same method

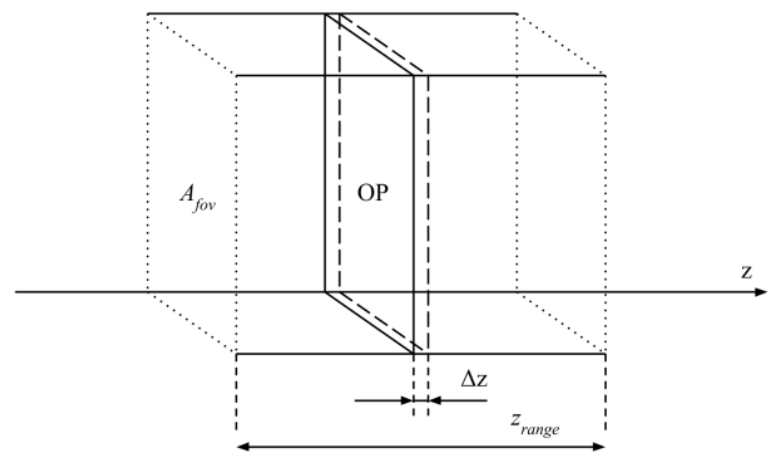

Fig. 5. The imaginary measurement volume confined by the area $A_{f o v}$ and the measuring range $z_{\text {range }} . A_{f o v}$ is the visible area limited by the field of view. zrange is the average number of detection events during a linear translation of the object in the z-direction times the translation delta distance $\Delta z$. OP is the plane of the reference object.

as the one that will be used to measure droplets. Since the dots are of known size and are expected to give a similar shadowgraph image as the droplets, the results are translated into two calibration tables.

The first calibration table is a list of the mean deviation between measured and actual diameter. This table is interpolated and expressed as a function, or correction factor $f$ of the measured diameter, $d^{M}$. The true diameter, $d^{T}$, can be derived by subtracting the correction factor.

$$
d^{T}=d^{M}-f\left(d^{M}\right)
$$

The second calibration table is a list of the ranges in the distance from the camera where the droplets of a given size are expected to be detected with the chosen method. Given a method (camera, optics, and detection algorithm) this range, $z_{\text {range }}$, will depend on both the true diameter, $d^{T}$, and the signal to noise ratio $\mathrm{snr}_{j}$.

$$
z_{\text {range }}=g\left(d^{T}, \operatorname{snr}_{j}\right)
$$

Therefore, to estimate the measurement volume of a particle, the true diameter $d^{T}$, and the $\operatorname{snr}_{j}$, must first be calculated.

\section{G. Measurement Volume and Measuring Range}

Fig. 5 illustrates the imaginary volume. Since the camera lens is telecentric, the field of view defined by the area $A_{f o v}$ is approximately the same in all positions on the z-axis. The maximum telecentric error of the lens is 0.091 degrees at the corners. At the maximum measured distance from object plane, $1.5 \mathrm{~mm}$, the maximum lateral error would then be $1500 \sin 0.091=2.4 \mu \mathrm{m}$. On the area $A_{\text {fov }}$, this corresponds to an error of less than 0.17 percent. When an object is translated in fixed steps in the z-direction, the number of detections will define a range, $z_{\text {range }}$, that together with $A_{f o v}$ confines a measuring volume, $V_{i}$, for a particle $i$ of a given size.

$$
V_{i}=z_{\text {range }} A_{\text {fov }}
$$

This volume is the imaginary volume in which one particle with the same shadow image as the reference object, if present, 
should be detected on average. If $m$ calibration objects are detected when moved in steps of $\Delta z$ in the complete depth of field, the measuring range $z_{\text {range }}=m \Delta z$.

A boundary fill function was used to distinguish closed contours from not closed contours. The range is given by the number of closed contours from one or several image series within the range times the step size between each translation in the direction from the camera. Contours that are not closed will not be counted in the range.

\section{H. Liquid Water Content}

Each detected particle with mass $m_{j}$, has a corresponding expected detection volume, $V_{j}$, which is determined by the area of view and the range in focus where the particles of that size are expected to be detected.

The LWC from one image is given by the sum of all the $n$ detected particles' masses of that image, divided by their respective expected detection volumes in that image.

$$
L W C=\sum_{j=1}^{n} \frac{m_{j}}{V_{j}}
$$

If several images are made, the mean LWC is given by the sum divided by the number of images.

Using the method described to find droplets one may need to consider the case where two or more droplets are close enough to be interpreted as one. To deal with this possibility we use a threshold on the symmetry, the major axis divided by the minor axis, and the compactness [37], to exclude noncircular objects from the calculation. In Chapter IV, we discuss this further.

\section{Median Volume Diameter}

The median volume diameter, MVD, is given at the point where half of the total volume of liquid content in a fixed air volume consists of droplets with diameters larger and the other half has smaller diameters. Compared with the mean volume droplet diameter and the mean droplet diameter, the MVD has been shown to give the best approximation to the mean collision efficiency of supercooled droplets [38].

To get the MVD from one image, we first calculate the LWC for that image. Then we sort all $\frac{m_{j}}{V_{j}}$ and count the index number $j$ until we get the sum LWC/2. The MVD is given by the mean of the last counted particle's diameter and the diameter of the next uncounted particle.

To average the MVD of several images we need to weigh the MVD with the LWC for each image. If we have $m$ images, and give the properties of each image the index $k$, the average MVD for all images is:

$$
\overline{M V D}=\frac{\sum_{k=1}^{m}\left(M V D_{k} L W C_{k}\right)}{\sum_{k=1}^{m} L W C_{k}} .
$$

\section{J. Measurement of Droplets in Air}

Before a measurement starts, the calibration tables $f$ and $g$ for size and volume determination are read. The tables are translated into calibration lookup tables using linear interpolation.

The image is captured and transferred from the camera or the disk memory, and edge detected using an edge detection algorithm.

After the edge detection, objects are qualified as droplets, and an uncorrected diameter is determined for each of these. For each detected droplet $j$ the signal to noise ratio, $\operatorname{snr}_{j}$, is determined.

Given the measured diameter $d^{M}$ of every particle, a corrected true diameter $d^{T}$ is calculated by subtracting the corresponding value in the calibration lookup table.

Using the true diameter, $d^{T}$, the particle's volume and mass is calculated. Its mass, $m_{j}$, is determined from the density of water, which in this study is approximated to $\rho=1 \mathrm{~g} / \mathrm{cm}^{3}$.

LWC and MVD are calculated using the data from one or several images.

\section{K. Investigation of Ambient Light}

The level of ambient light in the optics lab was too low to give any contribution to the exposure during the experiment. Therefore, an experiment was carried out outside in daylight. An ultrasonic fog generator was used to simulate a fog spreading the ambient light into the telecentric lens. The generator was set on maximum power. Two measurements (1 and 2) were done in semi-sunny conditions, and two measurements (3 and 4) were done in direct sunlight.

The shortest possible exposure time according to the camera specification is $0.038 \mathrm{~ms}$, slightly depending on other camera settings. For each measurement 152 images were captured, with increasing exposure time from 0.04 to $2.0 \mathrm{~ms}$. A delay of 1 second was set between each image.

\section{Light Intensity Levels}

To analyze the effect of different signal to noise ratios, the flashlight intensity, and camera gain settings were adjusted to make eight different exposure cases. Since the camera had fixed levels for the master and the analogue gain, the flashlight energy levels were adjusted from these gain levels to achieve similar image gray scale levels for all cases. The duration time of flashes, and the driving currents of the LED, were altered until the maximum gray level of any pixel was high but well below the saturation point; approximately 90 percent of the maximum in 12-bit mode. The gain levels could be adjusted by changing either "analogue gain" between -10 and 4 or "master gain" to $100,200,300$ or 400 . The eight cases are labeled G-10MG100, G-5MG100, G0MG100, G2MG100, G4MG100, G4MG200, G4MG300 and G4MG400.

The light energy at each exposure level was investigated using a thermal power sensor (Thorlabs S302C) and a pulsed signal, 3-10 $\mu \mathrm{s}, 9-36 \mathrm{kHz}$. To reduce the thermal effect of the heated glass and the ambient light, the power was measured immediately before and after start and stop. The pulse length was measured using a semiconductor light sensor connected to an oscilloscope. The result is shown in Fig. 9. 


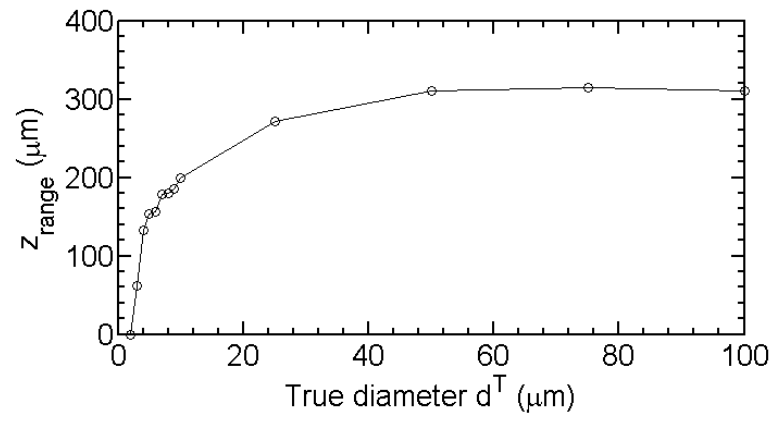

Fig. 6. Results of measuring range zrange depending on true diameter using Laplacian of Gaussian $13 \times 13$ and threshold of 0.002 applied on dots of $2,3,4,5,6,7,8,9,10,25,50,75$ and $100 \mu$ m diameter in one exposure case.

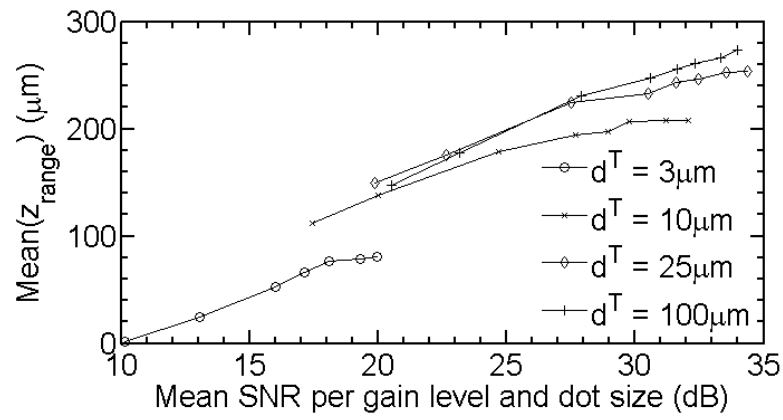

Fig. 7. Results of measuring range $z_{\text {range }}$ depending on mean $\operatorname{snr}_{j}$ using Laplacian of Gaussian $13 \times 13$ and threshold of 0.002 applied on dots of 3, 10, 25 and $100 \mu \mathrm{m}$ diameter with eight different exposure cases. Every marker is the mean $z_{\text {range }}$ of 100 series of images.

\section{RESULTS}

\section{A. Estimating Measuring Range}

The measuring range $z_{\text {range }}$ was first determined for one exposure case, G0MG100, by measuring the dots of all available sizes in the range covered by the optical focus. It is determined by the number of detected dots times the step length between each measurement. The result of this measurement is presented in Fig. 6 .

Fig. 7 shows the mean measuring range mean $\left(z_{\text {range }}\right)$ depending on $\mathrm{snr}_{j}$ for multiple measurements of the dots with true diameters, $d^{T}=3,10,25$ and $100 \mu \mathrm{m}$. For each of the eight exposure cases, the dots were measured 100 times in range steps of two micrometers, resulting in 80,000 images for the $3 \mu \mathrm{m}$ dot, and 160,000 images each for the other three sizes. Images creating a closed contour from the Laplacian of Gaussian edge detection, threshold 0.002 are counted. As each step was two micrometers, the measuring range (in micrometers) is the number of detected dots within the range times two.

Fig. 8 shows the measuring range coefficient of variation $C V\left(z_{\text {range }}\right)$ for the 100 measuring ranges, rng, calculated for each SNR case.

$C V\left(z_{\text {range }}\right)(s n r)=\frac{\sum_{\text {rng=1 }}^{100}\left(z_{\text {range }}-\text { mean }\left(z_{\text {range }}\right)\right)^{2}}{\text { mean }\left(z_{\text {range }}\right)}$

The coefficient of variation is the ratio of the standard deviation to the mean. The standard deviation decreases with increasing SNR.

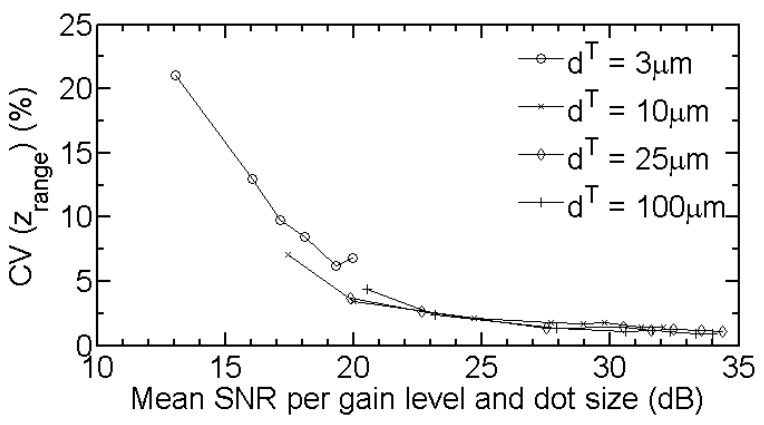

Fig. 8. Coefficient of variation for the mean measuring range.

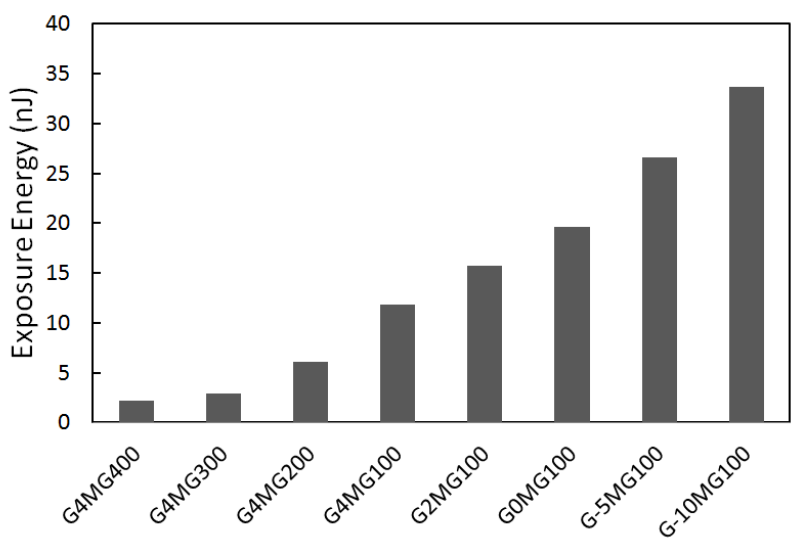

Fig. 9. Light energy (nJ) for exposure in each of the eight gain levels used. The energy was tuned manually up to a level of high exposure, but not saturating any point in the image.

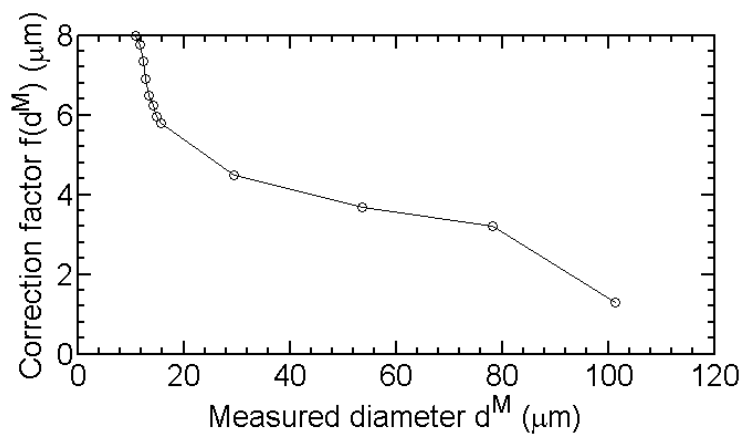

Fig. 10. Difference between measured and actual diameter, $f\left(d^{M}\right)$, using the Laplacian of Gaussian $13 \times 13$ with threshold 0.002 to define the measured area by edge detection.

\section{B. Estimating True Diameter}

The difference between the measured and the true diameter from the micrometer scale was used to create the lookup table for size calibration. Fig. 10 illustrates the correction factor $f\left(d^{M}\right)$ using the Laplacian of Gaussian $13 \times 13$ with threshold 0.002 to define the measured area by edge detection.

Fig. 11 shows the coefficient of variation $C V\left(d^{M}\right)$ for the measured diameter $d^{M}$ when measured on the 3,10 , 25 and $100 \mu \mathrm{m}$ dots in eight different exposure cases. For the three micrometer dot, the two lowest exposure levels did not result in a measurement. The other decreased from 0.72 percent (G4MG200, SNR 13dB) to 0.22 percent (G-10MG100, SNR 20dB). The variation coefficient of the 


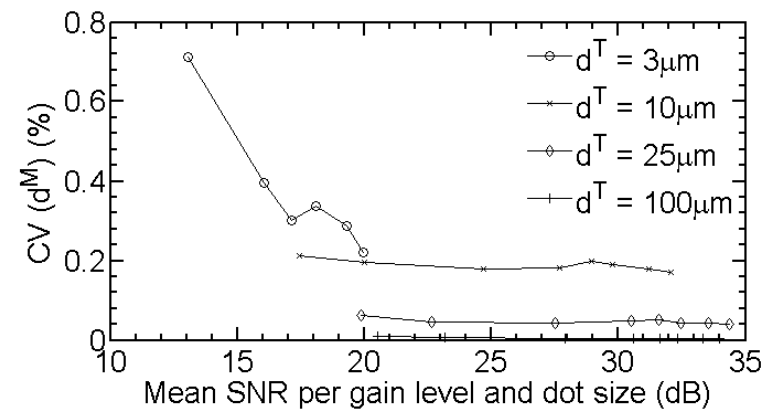

Fig. 11. Coefficient of variation from 100 series of measured diameters $d^{M}$ for the $3,10,25$ and $100 \mu \mathrm{m}$ dots.

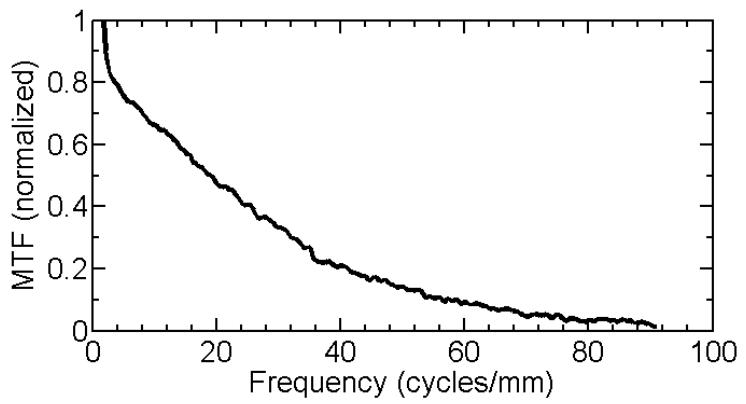

Fig. 12. MTF curve of the system measured at optimal focus by using FFT analysis of the gradient of the step response for a sharp edge in the center of the field of view. Frequency is given in sinus cycles per $\mathrm{mm}$ and the contrast is normalized between the darkest and lightest parts of the image.

ten micrometer dot decreased from 0.22 to 0.17 percent. The variation coefficient of the $25 \mu \mathrm{m}$ dot varied between 0.063 and 0.044 percent and for the $100 \mu \mathrm{m}$ dot it varied between 0.0085 and 0.0032 percent.

\section{Energy Required for Exposure}

The illumination energy required to get a good exposure for each of the eight gain setting levels is shown in Fig. 9. $34 \mathrm{~nJ}$ is required for the area in view $\left(7.8 \mathrm{~mm}^{2}\right)$ for the lowest gain setting, which results in the highest SNR. The area in view is about 8 times smaller than the illuminated area of the collimated LED, which was measured to about $8 \times 8 \mathrm{~mm}$.

\section{Measuring the Optical Transfer Function}

The MTF curve was measured using FFT (Fast Fourier Transform) analysis in Matlab on the gradient (impulse response) of images of a straight edge on the micrometer scale. The micrometer scale was moved in steps of five micrometers in z-direction, creating one MTF curve for every step. The result from one position (at optimal focus) is shown in Fig. 12, and the resolution limit at 30 percent contrast depending on the distance from optimal focus is shown in Fig. 14.

\section{Discussion And ANALYsis}

\section{A. The Optical System Limitations}

The optical resolution of the system is limited by the wavelength of the illuminating light, the lens resolution and the spatial resolution of the sensor.

The number of detected droplets per image depends on the density of the droplet distribution, and a less dense fog will require a larger ensemble of images to measure equally many droplets. Therefore we sought a camera with not only high sensitivity and high signal to noise ratio, but also high imaging rate and transfer speed. Global shutter is essential since the droplets in reality are moving and the exposure only occurs during the short illuminating flash. The frame rate is limited by the achievable transfer rate from the camera to the analyzing computer and the analyzing time for every image.

The $5.5 \mu \mathrm{m}$ pixels of the CMV4000 sensor are equipped with micro lenses, raising the efficiency from 42 percent (fill factor without micro lens) to 60 percent (quantum efficiency times fill factor at $550 \mathrm{~nm}$ ) according to the manufacturer's data sheet. The micro lenses would also mean a smaller risk for digital aliasing. Spatial resolution of a digital image system can be given in the unit of line pairs per $\mathrm{mm}(\mathrm{lp} / \mathrm{mm})$. A $5.5 \mu \mathrm{m}$ pixel pitch means that the finest resolvable frequency at the focal plane according to Nyquist-Shannon sampling theorem is $1 /(2 \cdot 0.0055)=91$ line pairs $/ \mathrm{mm}(\mathrm{lp} / \mathrm{mm})$.

A telecentric lens simplifies measuring as the magnification is constant with varying distance to the object. The TC4M004-C has a magnification of $4 \times$ and a working $\mathrm{f} / \#$ of 22. A similar lens with lower magnification but with the same working $\mathrm{f} / \#$ would increase the depth of field, thus creating a larger measuring volume. Since the depth of field is approximately inversely proportional to the square of the magnification, a similar lens with $2 \times$ magnification would give a $16 \times$ larger measuring volume for every image, albeit on the cost of resolution.

Rayleigh's criterion can be used as an estimation of the smallest resolvable distance in the object plane, $\Delta 1$.

$$
\Delta l \approx 1.22 \lambda \frac{1}{1+|m|} w F / \#
$$

With $\mathrm{wF} / \#=22, \lambda=455 \mathrm{~nm}$, and $\mathrm{m}=4$ we get $\Delta \mathrm{l}=$ $2.44 \mu \mathrm{m}$. In the focal plane, with $4 \times$ magnification, this would correspond to a theoretical maximum diffraction limited resolution of $(1 / 0.00244) / 4=102 \mathrm{lp} / \mathrm{mm}$. Due to imperfections in the lens design and manufacturing, the usable resolution is less, especially closer to the sensor edges. Compare with the system MTF measured at optimal focus shown in Fig. 12. This curve indicates a resolution of only about $58 \mathrm{lp} / \mathrm{mm}$ at 10 percent contrast.

Using a high power LED instead of laser reduces the interference effects used in e.g. holography [28], but since it is a monochromatic source, interference may not be completely ruled out. The coherence length, provided a Gaussian spectrum, can be approximated by Eq. 12 [39]:

$$
l_{c} \approx \sqrt{\frac{2 \ln 2}{\pi}} \frac{\lambda_{0}^{2}}{\Delta \lambda}
$$

$\Delta \lambda$ is the $-3 \mathrm{~dB}$ spectral bandwidth and was measured to $20 \mathrm{~nm}$ for the blue LED used in this study. The actual peak was measured to $453 \mathrm{~nm}$. Applying this in Eq. 12 we estimate the coherence length to about $6.8 \mu \mathrm{m}$.

LEDs can sometimes be used with currents far above the specifications, as long as the pulse length is short and the duty cycle is low enough to permit the heat generated to be transported away between the pulses. Using the LED above 


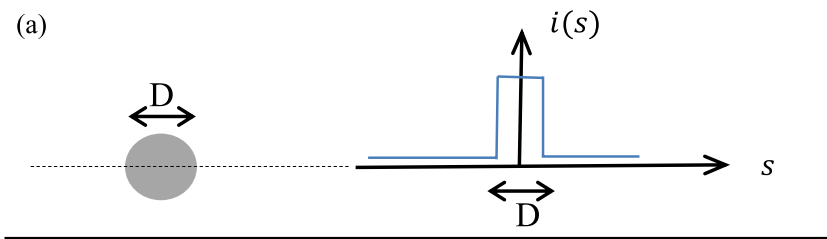

(b)

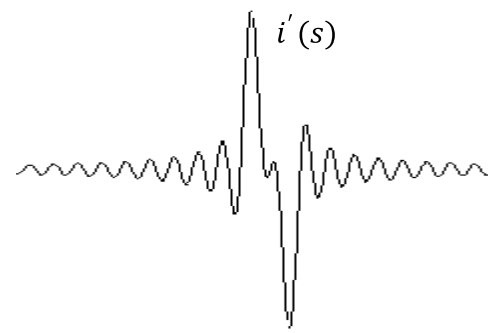

Fig. 13. (a) Shows a simplification of an imaged particle with diameter $\mathrm{D}$ as a square pulse in one dimension. (b) Shows the first derivate $i^{\prime}(s)$ achieved by assuming the lens works as a low pass filter in the frequency domain.

specifications may though affect the efficiency and aging of the LED. LED emittance also depends on the temperature. Depending on the capacitance of the diode, the rise time may limit the current, although there exist some techniques to shorten the LED pulses [40], [41].

\section{B. Laplacian and Boundary Fill as Selection Criteria}

In this study we use the Laplacian of Gaussian for edge detection and noise filter. In brief comparison with other edge detection algorithms, like Canny [42], it gives the best result. This is not to say there is no room for optimization. We define the edge where the second derivate of the between zero and one normalized image is above 0.002 . Lowering the threshold to 0.001 gives too much response from noise and raising the threshold reduces the measuring range.

The $13 \times 13$ size convolution kernel is a digital approximation of the true Laplacian of Gaussian function. Increasing the size of the kernel would mean more calculations for each processed image, and a smaller usable field of view, but could also lower the noise sensitivity.

Subtracting the edge detected binary image from the same image with binary fill applied, sorts out the boundaries that have closed contours. As this works as selection criteria for which droplets to count and which not to count, it will also define the measuring range. The precision of the measuring range is estimated by taking series of images of the same dot, and moving the dot a distance $\Delta \mathrm{z}$ between each image in every series. In each of the image series, we use the standard deviation as a measure of the precision. This was done for 100 series of images of the 3,10, 25 and $100 \mu \mathrm{m}$ dots.

As was shown in Section II., the Laplacian is a second order derivate. This means that a particle can be segmented as long as the edge of an imaged droplet has strong enough gradient, as shown in Fig. 2. To explain this we can imagine an imaged particle of size D where the intensity profile in one dimension $s$ is graphed as $i(s)$. See Fig. 13 (a). This is an ideal optical system having infinite resolution. The Fourier transform $F$ of $i(s)$ becomes $I(\omega)=F\{i(s)\}=\frac{2}{\omega} \sin \frac{\omega D}{2}$ where $\omega$ is the angular frequency. The strength of the first order

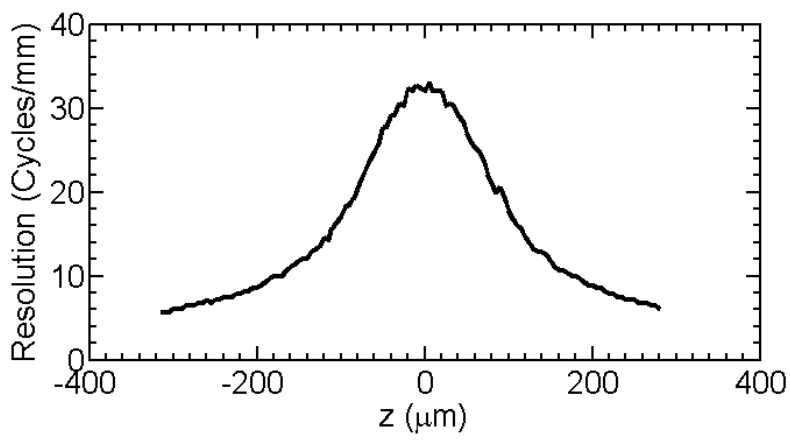

Fig. 14. Resolution limit at $30 \%$ contrast measured by FFT on an edge at different distances from the optimum focus.

gradient $\frac{d i(s)}{d s}=i^{\prime}(s)$ is assumed to give information about how sharp the droplet is imaged. The frequency transform of $i^{\prime}(s)$ is $I^{\prime}(\omega)=j \omega \cdot I(\omega)$. We now limit the resolution of the optical system to $\omega_{g}$. This limit allows computation of the first order gradient as the reverse Fourier transform $i^{\prime}(s)=$ $F^{-1}\left\{I^{\prime}(\omega)\right\}$ for $-\omega_{g}<\omega<\omega_{g}$. In this simplified analysis we are assuming that the lens system behaves as an ideal low pass filter having a limited angular frequency of $\omega_{g}$. We can estimate $\omega_{g} \approx \frac{2 \pi}{r}$ where $\mathrm{r}$ is the lens resolution in the focal plane by Rayleigh's criterion. The gradient can now be computed as:

$$
i^{\prime}(s)=\frac{\sin \left(\omega_{g}\left(s+\frac{D}{2}\right)\right)}{\pi\left(s+\frac{D}{2}\right)}-\frac{\sin \left(\omega_{g}\left(s-\frac{D}{2}\right)\right)}{\pi\left(s-\frac{D}{2}\right)}
$$

An example graph of $i^{\prime}(s)$ is shown in Fig. 13 (b). From this graph and Eq. 13 can be seen that maximum gradient strength $G_{\max }$ appears at $s= \pm \frac{D}{2}$ as long as D is large enough.

$$
G_{\text {max }}=i^{\prime}(s)=\lim _{S \rightarrow \pm \frac{D}{2}} i^{\prime}(s)= \pm \frac{\omega_{g}}{\pi}
$$

Small droplet sizes $D$ with respect to optical resolution $r$ will cause the two gradient peaks shown in Fig. 13 (b) to interfere. The maximum gradient strength $G_{\max }$ is proportional to the optical system resolution expressed as $1 \mathrm{p} / \mathrm{mm}$. We can also conclude from analyzing the MTF curve obtained empirically that larger distance from the optimal focus will result in lower resolution. Fig. 14 shows the resolution depending on the distance from the optimal focus, when the measured MTF is 30 percent. If we achieve a measuring range of $0.2 \mathrm{~mm}$ empirically by edge detecting our test objects, according to Fig. 14, we need a resolution of at least about $17-18$ cycles/mm to measure a particle. This also explains why we can use a gradient operator in combination with a threshold on gradient strength for the control of the measuring range.

\section{Comparing Drops With Dots}

In the study described in this paper we have assumed that a spherical object such as a water droplet in air will produce the same shadow image as a disc shaped dot printed on a sheet silicon glass.

The reason for making this assumption is first that despite the transparency, the refractive index of water makes the 
droplet work as a spherical lens that will scatter almost all of the light that reaches the droplet from different directions.

Some of the light will also be absorbed, albeit the absorption of a single water droplet is negligible due to its small volume and because water absorb very little in the visible spectrum. The combined effect of scattering and absorption is the extinction [29]. By using a telecentric lens for imaging, the amount of scattered light that will reach the camera is very small compared with the background illumination. This makes the droplet, despite being almost transparent, to appear as a black disc against the bright background.

Irregularities and dirt on the surface of the glass sheet will add uncertainty to the measurement of the reference object. The smallest dot measured, two micrometer in diameter, was even difficult to find as some disturbances close to it were equal in size or larger.

For larger droplets the light passing straight through the center of the drop is visible, and a function may be needed to handle this.

\section{Measuring Droplets}

A real distribution of water droplets was created by an ultrasonic fog generator. As they were not contained in a closed environment the droplets evaporated quickly after exiting the generator tube, making the true sizes difficult to verify. But from the calibration tables shown in Fig. 7 and Fig. 10 we can estimate the MVD and LWC for one sample image.

As mentioned previously, there may be cases where two droplets are close enough to appear as one much larger drop. A solution that was tried was to make a measurement of the droplet's circularity and add this as selection criteria. This solution may also work as a filter for ice or snow particles. Unfortunately this also reduces the measured number of particles, resulting in an underestimation of LWC. To deal with this there are at least a few alternatives, e.g. improving the circularity measurement [37].

Droplets that are very close are likely to coalesce, thereby decreasing the number concentration at a rate that appears to increase for larger droplets and more complex droplet size distributions [43]. Therefore we expect the likelihood of finding two droplets close to each other in a natural distribution to be low. A realistic fog with $\mathrm{LWC}=0.4 \mathrm{~g} / \mathrm{m}^{3}$ and droplet size five $\mu \mathrm{m}$ has the number concentration $6 \cdot 10^{9} \mathrm{~m}^{-3}$. With this concentration, only nine droplets, on average, are expected in a sample volume corresponding to one image with our system.

The accuracy and precision will depend on the number of droplets sampled. This means that for a population of droplets with large diameters, many more images are needed to get the same accuracy, than for a population of droplets with small diameters, given the populations have the same LWC.

\section{E. Particle Size}

The particle size is determined by the area bounded by the edge detection algorithm illustrated by the filled white blob in Fig. 2 (lower left). If a threshold is set relative to the background level that has a value below a given threshold limit, the measured area could be determined by the darkness of the object's shadow. This alternative could improve the accuracy of size for small droplets.

The size accuracy in general is limited by the resolution of the optical system and the effect of the diffracted light on the edge detection. In Fig. 11 one can see that the coefficient of variation for a $100 \mu \mathrm{m}$ dot is very low and that it increases with decreasing diameter. This effect is likely caused by the diffraction patterns. Although the optical resolution is too low to distinguish the diffraction patterns, it adds an uncertainty to the size measurement of small droplets.

The calibration is based on the mean measured size of any particle in the measuring range. Therefore the coefficient of variation in Fig. 11 is based on the measured diameter of all detected z-positions within the measuring range.

\section{F. Measuring Volume}

As can be seen in Fig. 7 and Fig. 8, the SNR is more important for small droplets. A high SNR gives a lower coefficient of variation for the measurement range and thus a more precise measurement of the LWC according to (11). It also means that the higher SNR we can achieve, the smaller the droplets we can find in the measuring range.

One should consider that the measurement volume is very small compared with the population of water particles in e.g. a cloud or a fog, and that the distribution of water may vary a lot in the physical surroundings. To get a good average value of the MVD and LWC, the number of counted particles needs to be large.

Sometimes the concentration of particles varies a lot within the same cloud or fog. Whether it is the average over a certain time, or the momentous maximum of LWC/MVD that is the most important to measure with regards to icing, future studies may reveal.

\section{G. LWC Precision}

We estimate the precision (coefficient of variation) of a measured value of the LWC based on two of the dots of known size $(10$ and $25 \mu \mathrm{m})$ of the micrometer scale. Here we assume that all the imagined measured droplets are of equal shape and have only one size and that the distribution is even. I.e. all $m_{j}$ and $V_{j}$ are equal for any $j$ so we skip the index $j$ for $m$ and $V$.

From this assumption and equation (7):

$$
\begin{aligned}
L W C & =n \frac{m}{V} \\
|\Delta L W C| & \leq n\left|\Delta \frac{m}{V}\right|
\end{aligned}
$$

Assuming that the relative errors of $m$ and $V$ are small and using the triangle inequality we get:

$$
\left|\frac{\Delta L W C}{L W C}\right| \lesssim\left|\frac{\Delta m}{m}\right|+\left|\frac{\Delta V}{V}\right|
$$

By calculating the mass of water using a density $\rho$ of $1 \mathrm{~g} / \mathrm{cm}^{3}$ we get the mass from the true diameter $d^{T}$ :

$$
m_{i}=\rho V_{i}=\frac{4 \pi\left(d^{T} / 2\right)^{3}}{3}=\frac{\pi}{6}\left(d^{T}\right)^{3}
$$


After logarithmic derivation we get:

$$
\left|\frac{\Delta m}{m}\right|=3\left|\frac{\Delta d^{T}}{d^{T}}\right|
$$

From Fig. 8 and Fig. 10 we read an approximate value of the variation coefficient for the case with $25 \mu \mathrm{m}$ droplets and SNR level $>27 \mathrm{~dB}$ :

$$
\begin{aligned}
\left|\frac{\Delta V}{V}\right|_{25 \mu \mathrm{m}} & <1.4 \% \\
\left|\frac{\Delta d^{T}}{d^{T}}\right|_{25 \mu \mathrm{m}} & <0.05 \% \\
\left|\frac{\Delta L W C}{L W C}\right|_{25 \mu \mathrm{m}} & <1.4 \%+3 \times 0.05 \%=1.6 \%
\end{aligned}
$$

The same calculation for $10 \mu \mathrm{m}$ droplets, again at an exposure case with SNR $>27 \mathrm{~dB}$, gives a larger statistical error:

$$
\begin{aligned}
\left|\frac{\Delta V}{V}\right|_{10 \mu \mathrm{m}} & <1.8 \% \\
\left|\frac{\Delta d^{T}}{d^{T}}\right|_{10 \mu \mathrm{m}} & <0.2 \% \\
\left|\frac{\Delta L W C}{L W C}\right|_{10 \mu \mathrm{m}} & <1.8 \%+3 \times 0.2 \%=2.4 \%
\end{aligned}
$$

The larger the statistical mass, i.e. the number of droplets measured, the closer the measured variations should be to the calculated variations. These calculations may not reveal the effect of digital aliasing, which would be higher for smaller droplets.

\section{H. Illumination Power Required at High Wind Speed}

Since the instrument is intended to image droplets moving with the air, a shorter and stronger illumination pulse than the one in the laboratory is required. From the results we see that we get the highest SNR at the highest energy, 34 nJ. To get a good image of a droplet only a few micrometers in diameter, it should move as little as possible during the exposure. As the risk of icing increases with increasing wind speed [44], it is important that the droplets are correctly measured also at higher wind speeds. Assuming that the highest possible wind speed is $50 \mathrm{~m} / \mathrm{s}$ and the droplet can move at most one micrometer for a correct measurement, the exposure time should be no more than $20 \mathrm{~ns}$. In this case, to get the highest SNR we need an average illumination power on the total area of view of $1.7 \mathrm{~W}$. But this power may be difficult to achieve with an LED.

The highest emitted power tested with the $455 \mathrm{~nm}$ Mightex LED was $348 \mathrm{~mW}$ at 2.5 Ampere driving current. As the collimated beam is about ten times ten millimeters, and the field of view is only three times three, only about one ninth of the emitted power is used for the exposure. This makes the system less sensitive to misalignment, but also quite inefficient. Since the area in view is nine times smaller than the collimated light beam this would mean $39 \mathrm{~mW}$ in the view area, which is about 44 times lower than the required, $1.7 \mathrm{~W}$ power for example above. But to make the light beam size smaller one would either have to use a lens of shorter focal length, which would lower the degree of collimation, or use a different light source with a smaller emissive area, which may not be possible with the required power. This is clearly a limitation of the LED when used to produce collimated light.

If we can use a lower SNR and still get useful images we would need less energy. If we e.g. set a higher camera gain (G4MG200 would require only $6 \mathrm{~nJ}$ of light energy), and if the wind speed can be reduced to $10 \mathrm{~m} / \mathrm{s}$, only $60 \mathrm{~mW}$ illumination power is required. In this case it should be possible to use the same LED to study droplets in a realistic environment.

Unfortunately, reducing the wind speed artificially by adding aerodynamic brakes will affect the air flow and this can change the properties of the measured particles [16]. It will also likely be more difficult to keep the instrument itself free from ice during the measuring.

\section{Choice of Camera, Lens and Illumination}

The camera is a c-mount CMOS camera with $2048 \times 2048$ resolution. The pixel size is $5.5 \mu \mathrm{m}$. Due to the strong commercial interest in this field, we expect this type of camera or similar to be available in large volumes and at even lower prices in the future. There are cameras with higher resolutions, but a higher resolution would not necessarily give better result since the lens resolution needs to match, and this would mean a higher system price. Smaller pixel sizes would also mean lower SNR, mainly due to the lower fill factor of the sensor. Lower SNR would then give a smaller measuring volume, following the results in Fig. 7 and Fig. 8.

The lens was chosen to fit the camera size and resolution. A lower magnification would increase the measuring volume, but raise the limit of the smallest particles possible to detect. A higher magnification puts higher requirements on the optics, resulting in a higher material cost.

Initially, two LED with different peak wavelengths (455 nm and $850 \mathrm{~nm}$ ) were tested, but since both the signal to noise ratio (SNR) and sharpness seemed better for the shorter wavelength, the $455 \mathrm{~nm}$ LED was used throughout the study. An $11 \mathrm{~mm}$ collimating lens was also tried. This lens is able to concentrate the beam more, but since the focusing distance becomes much shorter, which might cause a problem for the flow of particles, and the beam divergence is doubled, we chose only to use the $22 \mathrm{~mm}$ lens in the study.

\section{J. Ambient Light}

The background radiation was measured by imaging the generated fog in ambient daylight (22,000 lux) and (for a worst case scenario) in direct sunlight (80,000 lux). The result shows that unless the camera is pointed directly at the sun, an exposure time less than $0.2 \mathrm{~ms}$ will give no significant effect in daylight.

\section{CONCLUSIONS}

LWC and MVD can be derived from digital images of the droplets by using a shadowgraph imaging system with a collimated LED and a telecentric lens.

By comparing with dots of known sizes printed on a glass plate, we predict the LWC coefficient of variation to be less 
than 1.6 percent for droplets $25 \mu \mathrm{m}$ in diameter, and less than 2.4 percent for droplets $10 \mu \mathrm{m}$ in diameter. This error increases with decreasing droplet diameter.

A comparative study of the system with a reference instrument on a larger homogenous aerosol will show if the accuracy is good enough to predict icing.

\section{REFERENCES}

[1] N. Dalili, A. Edrisy, and R. Carriveau, "A review of surface engineering issues critical to wind turbine performance," Renew. Sustain. Energy Rev., vol. 13, pp. 428-438, Feb. 2009.

[2] S. Fikke et al., "COST 727: Atmospheric icing on structures," Meas. Data collection Icing, State Art, Publication MeteoSwiss, vol. 75, no. 110 , pp. 1381-1422, 2006.

[3] M. C. Homola, M. S. Virk, P. J. Nicklasson, and P. A. Sundsbø, "Performance losses due to ice accretion for a $5 \mathrm{MW}$ wind turbine," Wind Energy, vol. 15, no. 3, pp. 379-389, 2012.

[4] W. J. Jasinski, S. C. Noe, M. S. Selig, and M. B. Bragg, "Wind turbine performance under icing conditions," J. Solar Energy Eng., vol. 120, no. 1 , pp. 60-65, 1998.

[5] L. Makkonen, T. Laakso, M. Marjaniemi, and K. Finstad, "Modelling and prevention of ice accretion on wind turbines," Wind Eng., vol. 25 , no. 1, pp. 3-21, 2001.

[6] J. Shin and T. H. Bond, "Results of an icing test on a NACA 0012 airfoil in the NASA Lewis icing research tunnel," Lewis Research Center, Cleveland, OH, USA, NASA Tech. Memo. 105374, AIAA-92-0647, prepared for the 30th Aerosp. Sci. Meeting Exhibit sponsored by the Amer. Inst. Aeronaut. Astronaut., Reno, NV, USA, Jan. 6-9, 1992.

[7] G. Thompson, B. E. Nygaard, L. Makkonen, and S. Dierer, "Using the weather research and forecasting (WRF) model to predict ground/structural icing," in Proc. 13th Int. Workshop Atmos. Icing Struct. METEOTEST, Andermatt, Switzerland, 2009, pp. 1-8.

[8] B. E. K. Nygaard, J. E. Kristjánsson, and L. Makkonen, "Prediction of in-cloud icing conditions at ground level using the WRF model," J. Appl. Meteorol. Climatol., vol. 50, pp. 2445-2459, Dec. 2011.

[9] O. Parent and A. Ilinca, "Anti-icing and de-icing techniques for wind turbines: Critical review," Cold Regions Sci. Technol., vol. 65, no. 1, pp. 88-96, 2011.

[10] L. Makkonen, "Analysis of rotating multicylinder data in measuring cloud-droplet size and liquid water content," J. Atmos. Ocean. Technol., vol. 9, pp. 258-263, Jun. 1992.

[11] W. R. Sand, W. A. Cooper, M. K. Politovich, and D. L. Veal, "Icing conditions encountered by a research aircraft," J. Climate Appl. Meteorol., vol. 23, pp. 1427-1440, 1984.

[12] S. G. Cober, G. A. Isaac, and J. W. Strapp, "Characterizations of aircraft icing environments that include supercooled large drops," J. Appl. Meteorol., vol. 40, pp. 1984-2002, 2001.

[13] M. C. Homola, M. S. Virk, T. Wallenius, P. J. Nicklasson, and P. A. Sundsb $\varnothing$, "Effect of atmospheric temperature and droplet size variation on ice accretion of wind turbine blades," J. Wind Eng. Ind. Aerodyn., vol. 98, no. 12, pp. 724-729, 2010.

[14] R. F. Ide, Comparison of Liquid Water Content Measurement Technquies in an Icing Wind Tunnel, document NASA/TM-1999-209643, ARL-TR2134, U.S. Army Res. Lab., Glenn Res. Center, Cleveland, OH, USA 1999.

[15] D. Baumgardner, "An analysis and comparison of five water droplet measuring instruments," J. Climate Appl. Meteorol., vol. 22, pp. 891-910, May 1983.

[16] D. Baumgardner et al., "Airborne instruments to measure atmospheric aerosol particles, clouds and radiation: A cook's tour of mature and emerging technology," Atmos. Res., vol. 102, pp. 10-29, Oct. 2011.

[17] P. Kulkarni, P. A. Baron, and K. Willeke, Eds., Aerosol Measurement: Principles, Techniques, and Applications. New York, NY, USA: Wiley, 2011.

[18] M. C. Homola, P. J. Nicklasson, and P. A. Sundsbø, "Ice sensors for wind turbines," Cold Regions Sci. Technol., vol. 46, no. 2, pp. 125-131, 2006.

[19] J. K. Spiegel, P. Zieger, N. Bukowiecki, E. Hammer, E. Weingartner, and W. Eugster, "Evaluating the capabilities and uncertainties of droplet measurements for the fog droplet spectrometer (FM-100)," Atmos. Meas. Techn., vol. 5, no. 9, pp. 2237-2260, 2012.

[20] R. D. Cohen, "Shattering of a liquid drop due to impact," in Proc., Math. Phys. Sci., vol. 435, no. 1895, pp. 483-503, 1991.
[21] A. V. Korolev, S. V. Kuznetsov, Y. E. Makarov, and V. S. Novikov, "Evaluation of measurements of particle size and sample area from optical array probes," J. Atmos. Ocean. Technol., vol. 8, pp. 514-522, Aug. 1991.

[22] M. Wendisch and J.-L. Brenguier, Eds., Airborne Measurements for Environmental Research: Methods and Instruments. Weinheim, Germany: Wiley, 2013.

[23] R. G. Knollenberg, "The optical array: An alternative to scattering or extinction for airborne particle size determination," J. Appl. Meteorol., vol. 9, pp. 86-103, Feb. 1970.

[24] J. E. Dye and D. Baumgardner, "Evaluation of the forward scattering spectrometer probe. Part I: Electronic and optical studies," J. Atmos. Ocean. Technol., vol. 1, pp. 329-344, Dec. 1984.

[25] G. Mie, "Beiträge zur Optik trüber Medien, speziell kolloidaler Metallösungen,” Ann. Phys., vol. 330, no. 3, pp. 377-445, 1908.

[26] D. Baumgardner, H. Jonsson, W. Dawson, D. O'Connor, and R. Newton, "The cloud, aerosol and precipitation spectrometer: A new instrument for cloud investigations," Atmos. Res., vols. 59-60, pp. 251-264, Oct./Dec. 2001

[27] R. P. Lawson and R. H. Cormack, "Theoretical design and preliminary tests of two new particle spectrometers for cloud microphysics research," Atmos. Res., vol. 35, pp. 315-348, Jan. 1995.

[28] J. Henneberger, J. P. Fugal, O. Stetzer, and U. Lohmann, "HOLIMO II: A digital holographic instrument for ground-based in-situ observations of microphysical properties of mixed-phase clouds," Atmos. Meas. Techn., vol. 6, no. 3, pp. 4183-4221, 2013.

[29] C. F. Bohren and D. R. Huffman, Absorption and Scattering of Light by Small Particles. New York, NY, USA: Wiley, 2008.

[30] A. D. Ward, M. Zhang, and O. Hunt, "Broadband Mie scattering from optically levitated aerosol droplets using a white LED," Opt. Exp., vol. 16, no. 21, pp. 16390-16403, 2008.

[31] J. Estevadeordal and L. Goss, "PIV with LED: Particle shadow velocimetry (PSV)," in Proc. 43rd AIAA Aerosp. Sciences Meeting Exhibit., 2005, pp. 12355-12364.

[32] C. Willert, B. Stasicki, J. Klinner, and S. Moessner, "Pulsed operation of high-power light emitting diodes for imaging flow velocimetry," Meas. Sci. Technol., vol. 21, no. 7, p. 075402, 2010.

[33] R. J. H. Klein-Douwel, P. J. M. Frijters, L. M. T. Somers, W. A. de Boer, and R. S. G. Baert, "Macroscopic diesel fuel spray shadowgraphy using high speed digital imaging in a high pressure cell," Fuel, vol. 86, nos. 12-13, pp. 1994-2007, 2007.

[34] P. J. Connolly, M. J. Flynn, Z. Ulanowski, T. W. Choularton, M. W. Gallagher, and K. N. Bower, "Calibration of the cloud particle imager probes using calibration beads and ice crystal analogs: The depth of field," J. Atmos. Ocean. Technol., vol. 24, pp. 1860-1879, Nov. 2007.

[35] D. Marr and E. Hildreth, "Theory of edge detection," Proc. Roy. Soc. London. B, Biol. Sci., vol. 207, pp. 187-217, Feb. 1980.

[36] R. C. Gonzalez and R. E. Woods, Digital Image Processing, 2nd ed. Englewood Cliffs, NJ, USA: Prentice-Hall, 2002.

[37] R. S. Montero and E. Bribiesca, "State of the art of compactness and circularity measures," Int. Math. Forum, vol. 4, no. 27, pp. 1305-1335, 2009.

[38] K. J. Finstad, E. P. Lozowski, and L. Makkonen, "On the median volume diameter approximation for droplet collision efficiency," J. Atmos. Sci., vol. 45, pp. 4008-4012, Dec. 1988.

[39] C. Akcay, P. Parrein, and J. P. Rolland, "Estimation of longitudinal resolution in optical coherence imaging," Appl. Opt., vol. 41, no. 25, pp. 5256-5262, 2002.

[40] O. Veledar, P. O. Byrne, S. Danaher, J. I. Allen, L. F. Thompson, and J. E. McMillan, "Simple techniques for generating nanosecond blue light pulses from light emitting diodes," Meas. Sci. Technol., vol. 18, no. 1, p. 131, 2007.

[41] H. Tanaka, Y. Umeda, and O. Takyu, "High-speed LED driver for visible light communications with drawing-out of remaining carrier," in Proc. IEEE Radio Wireless Symp. (RWS), Jan. 2011, pp. 295-298.

[42] J. Canny, "A computational approach to edge detection," IEEE Trans. Pattern Anal. Mach. Intell., vol. 8, no. 6, pp. 679-698, Nov. 1986.

[43] R. Bordás, T. Hagemeier, B. Wunderlich, and D. Thévenin, "Droplet collisions and interaction with the turbulent flow within a two-phase wind tunnel," Phys. Fluids, vol. 23, no. 8, p. 085105, 2011.

[44] L. Makkonen, "Models for the growth of rime, glaze, icicles and wet snow on structures," Philos. Trans. Roy. Soc. London A, Math. Phys. Sci., vol. 358, no. 1776, pp. 2913-2939, 2000. 


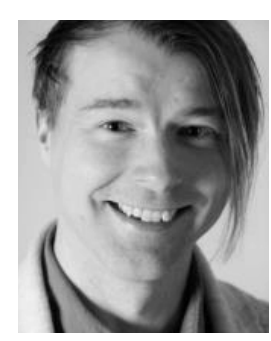

Staffan Rydblom received the M.S. degree in applied physics and electrical engineering from Linköping University, Sweden, in 2001. $\mathrm{He}$ is currently pursuing the Ph.D. degree with Mid Sweden University. From 2002 to 2009, he was with Bombardier Transportation, involved in the design of train control systems and engineering bid management. He did engineering project management at Permobil from 2009 to 2011, and was with ABB Service from 2012 to 2013.

His research interests include measuring techniques for atmospheric icing on structures and real-time image processing.

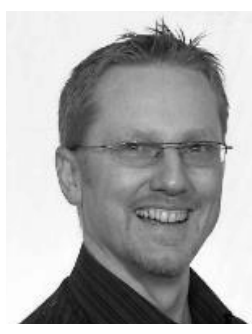

Benny Thörnberg received the degree in electronics design, the B.Sc. degree in electrical engineering, and the Licentiate(Tech.) and Ph.D. degrees from Mid Sweden University, Sundsvall, Sweden, in 1988, 2001, 2004, and 2006, respectively. He was with Regam Medical Systems AB, Sundsvall, from 1990 to 1997 , where he was involved in a camera design for intraoral X-ray imaging. He is currently an Assistant Professor with the Department of Electronics Design, Mid Sweden University. His current research interests include machine vision, metrology, design methods for embedded systems and, in particular, real-time video processing and analysis. 